\title{
Actualización y manejo clínico de los anticuerpos anti-ácido desoxirribonucleico
}

https://doi.org/10.1515/almed-2020-0067

Recibido 09-07-2020; aceptado 19-12-2020;

publicado en línea 03-02-2021

Resumen: Los anticuerpos contra el ácido desoxirribonucleico [DNA] en el laboratorio clínico, están íntimamente ligados al diagnóstico y monitorización del lupus eritematoso sistémico [LES]; no obstante, las características de los métodos analíticos y las propiedades de los propios anticuerpos son heterogéneas Revisar la definición y propiedades de los anticuerpos anti-DNA de doble cadena [antidsDNA], la adecuación de los métodos analíticos y los requerimientos clínicos para este biomarcador. A través de PubMed se investiga la bibliografía existente con los términos anti-dsDNA, editorial, review, guideline, meta-analysis y LES. La última búsqueda, anti-dsDNA y LES restringuida a los últimos dos años. Se amplía información a través de artículos relacionados y los publicados en organismos oficiales estatales relacionados con anti-dsDNA y LES. Se analizan los métodos del laboratorio clínico para el análisis de los antidsDNA y sus características. Se revisa la utilidad clínica de los anti-dsDNA en sus aspectos diagnóstico, de asociación clínica y seguimiento del LES. Existe una amplia variabilidad en los métodos analíticos y persisten déficits en la estandarización. Forman parte de los criterios actuales clasificatorios de LES y se utilizan como marcadores en el seguimiento de la enfermedad. La utilidad diagnóstica mejora cuando se determinan en pacientes con ANA positivos. En el seguimiento, es interesante la cuantificación, preferiblemente con el mismo método analítico (dado los déficits de estandarización).

\footnotetext{
*Autor para correspondencia: M. Belén Aparicio Hernández, Servicio Bioquímica y Análisis Clínicos, Complejo Asistencial Universitario Salamanca, Paseo San Vicente 58-182, 37007 Salamanca, España, E-mail: Salaautoinmunidad@yahoo.es

Concepción González Rodríguez, Unidad de Bioquímica Clínica, Hospital Universitario Virgen Macarena Sevilla, Sevilla, España Inmaculada Alarcón Torres, Servicio Análisis Clínicos, Hospital Universitario Gran Canaria-HUGCDN, Gran Canaria, España
}

Palabras clave: anti-dsDNA; marcador clínico; lupus eritematoso sistémico.

\section{Introducción}

La baja prevalencia y un espectro amplio de síntomas y signos dificultan el diagnóstico de las enfermedades reumáticas autoinmunes sistémicas. Por ello, se precisan biomarcadores, tales como, los anticuerpos dirigidos contra el ácido desoxiribonucléico [DNA] [1].

Los anticuerpos contra el DNA pueden reconocer todas las estructuras del DNA presentes en la cromatina, tanto en su estado de reposo como activo. Reconocen secuencias de DNA, DNA lineal de cadena sencilla, DNA de doble cadena [dsDNA]bien circular o bien helicoidalen sus diferentes formas: BdsDNA, la más habitual con la doble hélice girando a la derecha, $\mathrm{Z}$ dsDNA girando hacia la izquierda, dsDNA elongado con la hélice alargada y dsDNA superenrollado y girando sobre sí mismo [2]. Sin embargo, no se han demostrado asociaciones clínicas para las subpoblaciones de anti-dsDNA dirigidas contra las estructuras descritas de anti-dsDNA [2]. En esta revisión se considerarán incluidas en el término general de anti-dsDNA. Por otro lado, los anti-ADNdc pueden ser de clase IgA, IgG e Ig M; pero los segundos son los más relevantes clínicamente y los que habitualmente se determinan en la práctica clínica [3].

El espectro de métodos analíticospara la determinación de anti-dsDNA es muy amplio y con diferentes características; lo que dificulta su utilización y significado clínico. Por ello, el objetivo de este trabajo se centra en revisar la definición y propiedades de los anticuerpos antidsDNA, analizar las propiedades analíticas y clínicas de los métodos de medida y aportar herramientas que faciliten el empleode anti-dsDNA como biomarcador en la atención del paciente con LES.

\section{Materiales y métodos}

Se realizan varias investigaciones bibliográficas avanzadas en PubMed con los términos anti-dsDNA y tipo de publicación; 
considerando editorial, review, guideline o meta-analysis. Igualmente, se realiza una búsqueda restringida a los últimos dos años con los términos anti-dsDNA y SLE. Se amplía información a través de la búsqueda de artículos relacionados y material publicado en organismos oficiales estatales relacionados con anti-dsDNA y LES.

\section{Resultados}

Se estructura la información revisada en cinco epígrafes: definición de anticuerpos anti-DNA y anti-dsDNA, propiedades, determinación, utilidad clínica y orientaciones de uso.

\section{Definición}

El DNA constituye el principal componente del material genético y es el responsable de almacenar la información genética. En las células procariotas y eucariotas se empaqueta en una estructura denominada nucleosoma; constituida por DNA e histonas [4].

Anticuerpos anti-DNA son aquellos que reconocen a las diferentes estructuras o componentes del DNA. Sin embargo, desde el punto de vista clínico y a pesar de sus limitaciones, el más relevante es el anti-dsDNA [5].

Los pacientes con LES generan anticuerpos contra dsDNA, bien aislado, unido a proteínas (ej. histonas) o integrado en estructuras más complejas como los nucleosomas [4].

Los anticuerpos contra los nucleosomas y las histonas poseen ciertas implicaciones clínicas. Mientras que los anti-histonas se asocian a lupus inducido por droga; los anti-nucleosomas, poseen un significado clínico similar a los anti-dsDNA, pudiendo detectarse en fases tempranas del LES, con una implantación clínica es muy inferior $[6,7]$.

\section{Propiedades}

Cuando se inicia una respuesta inmune humoral la afinidad de los anticuerpos es baja; pero conforme progresa, la afinidad del anticuerpo hacia su ligando [epítopo] se incrementa. En moléculas polivalentes, como son las inmunoglobulinas, se va incrementando, igualmente, la fuerza global con la que la molécula de inmunoglobulina interacciona con la molécula antigénica, a través de sus diferentes ligandos [avidez] [8]. En los centros germinales, se produce maduración y selección de la respuesta inmunológica que se manifiesta en las formas peptídicas de la región variable de las inmunoglobulinas [9].

En un contexto inmunogénico normal, un estímulo, como puede ser una infección bacteriana o vírica, es capaz de inducir una respuesta inmunológica transitoria de defensa que reconozca al anti-dsDNA de la bacteria o del virus e incluso del propio huésped [Figura 1a]. Si el estímulo persiste, los anti-dsDNA persisten a concentraciones bajas [Figura 1b]. Si el estímulo es muy fuerte la respuesta transitoria es fuerte igualmente [Figura 1c]. Pero en un contexto autoinmune, la respuesta anti-dsDNA reconoce al anti-dsDNA propio y se mantiene y progresa; incrementándose las concentraciones, al igual que la afinidad y avidez. Esta última reacción es la más característica y específica del LES [Figura 1d] [10].

Junto al agente infeccioso, otros estímulos [apoptosis exacerbada, exposición a radiaciones ultravioleta, fármacos...] pueden generar anti-dsDNA en pacientes con LES, cáncer, $u$ otras enfermedades autoinmunes [10-12]. Parece que los anti-dsDNA reconocenlas moléculas de DNA dañado con mayor afinidad y formando complejos inmunes más fuertes [13].

\section{Determinación}

\section{Métodos}

Hasta hace pocos años los anticuerpos anti-dsDNA se han detectado mediante métodos inmunoenzimáticos de ELISA [enzyme linked immunosorbent assay] [14], inmunofluorescencia indirecta sobre el hemoflagelado Crithidea

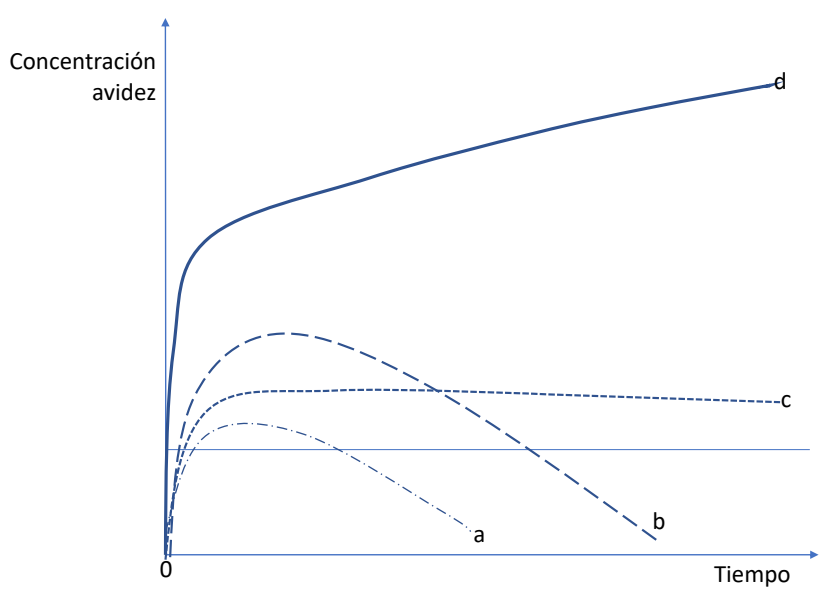

Figura 1: Perfiles teóricos de respuesta anti-dsDNA. (a) Uun estímulo ya sea una infección o autólogo origina una respuesta transitoria con concentraciones y avidez bajas de antidsDNA si es breve; (b) una respuesta transitoria pero más elevada cuando el estímulo es más fuerte; (c) unas concentraciones bajas pero persistentes de anti-dsDNA cuando el estímulo permanece; (d) una respuesta inmunológica con producción mantenida de antidsDNA a concentraciones y grado de avidez elevados en un contexto autoinmune adecuado. Modificada de Rekvig [10]. 
Luciliae [CLIFT] [15] y radioinmunoanálisis [16]; pero en las últimas décadas han surgido nuevos métodos como los inmunoanálisis enzimáticos fluorescentes [FEIA] [17], quimioluminiscentes [18] y los que analizan múltiples parámetros [anticuerpos] de forma simultánea [MPIA] [19, 20].

Los más utilizados en nuestro entorno son los métodos de FEIA y CLIFT, seguidos de EIA, CLIA y MPIA, de acuerdo a los informes metodológicos del programa de calidad UK-NEQAS que cuenta con más de 600 participantes [21]. Muy pocos laboratorios emplean inmunoblot o radioinmunoanálisis; ya que el primero no se recomienda [22] y el segundo ha sido sustituido por métodos que evitan el uso de radioisótopos [23].

La técnica de Farr, descrita en 1969 [24] ha sido considerada el método de referencia. Se caracteriza por ser cuantitativa y de especificidad elevada para el LES. La especificidad se atribuye al uso de altas concentraciones de sales [sulfato amónico] durante el paso de precipitación de los inmunocomplejos dsDNA/anti-dsDNA, lo que se cree, selecciona los anticuerpos de alta avidez [25]. Probablemente esta prueba posea la mejor relación sensibilidad/ especificidad para el LES; pero usa radioisótopos y detecta anti-dsDNA de clase IgA, IgG e IgM sin poder diferenciar entre ellas. Recientemente, se ha descrito un método de Farr modificado que intercala un colorante fluorescente en la molécula dsDNA (Farr-FIA). Se correlaciona muy bien con el método de Farrradioisotópico y alcanza una sensibilidad y especificidad diagnósticas del 53\% y 100\%, respectivamente [26].

En la CLIFT el dsDNA nativo está altamente compactado en el quinetoplasto. Posee especificidad elevada con alto valor predictivo positivo; pero su sensibilidad es inferior a la de otros métodos [especialmente en LES temprano] [27].

En la década de los 80, comienza a utilizarse métodos totalmente automatizados, primero ELISA y después FEIA, MPIA y CLIA. Estos métodos poseen, en general, mayor sensibilidad y menor especificidad que CLIFT [detectando anti-dsDNA de afinidad y avidez inferiores]; aunque las características varían mucho entre los fabricantes. Algunos de ellos utilizan concentraciones elevadas de sales en el tampón de lavado, tanto en ELISA [28] como en CLIA [29]; habiéndose descrito, en el último, buenas relaciones sensibilidad/especificidad [18].

En los últimos años, se ha descrito un método de detección fluorimétrica que permite la cuantificación de antidsDNA libre e integrado en complejos inmunes circulantes (DNA endógeno-antidsDNA); incorporándolos últimos, a la detección y cuantificación habitual [30].

\section{Características}

Las características de los métodos comerciales más habituales aparecen reflejadas en la Tabla 1; habiéndose referenciado cada método al equipo más utilizado por los laboratorios que participan en el programa de calidad UKNEQAS: Orgentec [EIA], Phadia 250 [FEIA], INOVA Quanta Flash [CLIA] y LuminextechnologyBioRadBioplex2200 [MPIA] [21].

Con respecto al sustrato, CLIA [18, 31] y MPIA [19] utilizan dsDNA sintético unido a partículas magnéticas o paramagnéticas. FEIA [17] y EIA [14], emplean dsDNA sintético o purificado unido a micro pocillos. En CLIFT, el dsDNA está expuesto en el quinetoplasto del hemoflagelado crithidia luciliae [15].

El conjugado, en general, permite la detección de anticuerpos de clase IgG; si bien hay excepciones, por ejemplo, algunos equipos de EIA detectan anti-dsDNA de clase IgG e IgM [Kallestad $\left.{ }^{\mathrm{TM}}\right]$ [32] y otros, permiten la detección de antidsDNA de clase IgA, IgG e IgM [Orgentec] [33].

La Tabla 1 muestra otras características de los métodos de análisis más frecuentes en los laboratorios clínicos europeos, de acuerdo a UK-NEQAS, información suministrada por los proveedores los reactivos e Infantino y cols. [33]. A continuación, se comentan algunas de las características:

- los tiempos de análisis son más largos en las técnicas de EIA y FEIA que en las de MPIA y CLIA. Cuando CLIFT, se realiza manualmente, tarda aproximadamente $60 \mathrm{~min}$ utos; si bien, este tiempo es variable dependiendo de la automatización y la lectura al microscopio de fluorescencia.

- los métodos FEIA, MPIA y CLIA permiten una carga continua de muestras; mientras que EIA y CLIFT trabajan en lotes.

- las curvas de calibrado son estables durante un periodo de tiempo variable en FEIA, MPIA y CLIA; mientras que en EIA debe realizarse una curva por lote de muestras y en CLIFT no existe curva de calibrado [en cada lote se realiza un control negativo y positivo].

- la detección es cuantitativa en todos los métodos excepto en CLITF, que es semicuantitativa con diluciones seriadas de las muestras. Aunque, en cualquiera de los métodos empleados, la expresión de resultados puede reducirse a cualitativa [positivo/negativo] utilizando un punto de corte establecido por el propio laboratorio o por el proveedor.

- el rango analítico varía entre los diferentes proveedores; siendo más amplio, en general, en los métodos de CLIA. 
Tabla 1: Características de diferentes métodos analíticos para anti-dsDNA.

\begin{tabular}{|c|c|c|c|c|c|c|c|c|}
\hline Método & Antígeno & Fase sólida & Conjugado & $\begin{array}{c}\text { Tiempo } \\
\text { análisis }\end{array}$ & Detección & Calibración & $\begin{array}{r}\text { Rango } \\
\text { analítico }\end{array}$ & Puntos corte \\
\hline CLIA & $\begin{array}{l}\text { dsDNA } \\
\text { sintético }\end{array}$ & $\begin{array}{l}\text { Bola para } \\
\text { magnética }\end{array}$ & $\lg G$ & $30 \mathrm{~min}$ & Cuantitativa & Curva & $9.8-666.9 \mathrm{UI} / \mathrm{mL}$ & $\begin{array}{r}35-45 \mathrm{UI} / \mathrm{mL} \\
\text { equívoco }>45 \mathrm{IU} / \mathrm{mL} \\
\text { positivo }\end{array}$ \\
\hline MPIA & $\begin{array}{l}\text { dsDNA } \\
\text { sintético }\end{array}$ & $\begin{array}{l}\text { Bola magnética } \\
\text { coloreada }\end{array}$ & $\lg G$ & $45 \mathrm{~min}$ & Cuantitativa & Curva & $1-300 \mathrm{Ul} / \mathrm{mL}$ & $\begin{array}{l}5-9 \mathrm{UI} / \mathrm{mL} \text { dudoso } \\
\geq 10 \mathrm{UI} / \mathrm{mL} \text { positivo }\end{array}$ \\
\hline FEIA & $\begin{array}{l}\text { dsDNA } \\
\text { sintético }\end{array}$ & Micro pocillo & $\lg G$ & $120 \mathrm{~min}$ & Cuantitativa & Curva & 0,5-379 UI/mL & $\begin{array}{l}10-15 \mathrm{UI} / \mathrm{mL} \text { dudoso } \\
>15 \mathrm{Ul} / \mathrm{mL} \text { positivo }\end{array}$ \\
\hline EIA & $\begin{array}{l}\text { dsDNA } \\
\text { sintético }\end{array}$ & Micro pocillo & $\lg G$ & $120 \mathrm{~min}$ & Cuantitativa & Curva & $0-200 \mathrm{UI} / \mathrm{mL}$ & $20 \mathrm{UI} / \mathrm{mL}$ \\
\hline IFI & $\begin{array}{l}\text { dsDNA } \\
\text { nativo }\end{array}$ & CrithideaLucilliae & $\lg G$ & $60 \mathrm{~min}$ & Cualitativa & Ninguna & $\mathrm{N} / \mathrm{A}$ & $1 / 10$ \\
\hline
\end{tabular}

Modificada de Infantino M y cols. [33]

Por otra parte, los puntos de corte y valores de referencia deben ser establecidos en cada laboratorio. La tabla recoge valores de referencia provistos por el proveedor. Como puede observarse, a pesar de que todos los métodos se referencian a un estándar internacional, persiste variabilidad en puntos de corte y rangos de referencia; lo que demuestra defectos de estandarización.

El estudio comparativo de diferentes equipos entre los métodos recogidos en la tabla, ha demostrado un grado de acuerdo de moderado a sustancial entre ellos, con índices kappa comprendidos entre 0,47 y 0,68 ; sensibilidades clínicas para LES variables entre 5,7\% [CLIFT] y 33,3\% [EIA]; especificidades entre 89,8\% [MPIA] y 98,8\% [CLIFT]; razón de verosimilitud positiva entre 2,93 [MPIA] y 17,6 [CLIFT] y una razón negativa de verosimilitud poco significativa para cualquiera de los métodos que osciló entre 0,71 [EIA] y 0,96 [33].

Como era de esperar y de acuerdo a la experiencia existente, CLIFT es menos sensible, pero posee mayor especificidad y cuando es positiva, confiere al paciente la mayor probabilidad de poseer LES [razón de verosimilitud positiva más elevada] [34, 35].

Por ello, el Ministerio de Sanidad y Consumo Español recomienda la determinación de anticuerpos anti-dsDNA mediante CLIFT a dilución 1:10 en pacientes con anticuerpos antinucleares para el diagnóstico del LES [36]. Es posible, utilizar CFLIT como técnica de segunda línea, método confirmatorio, después de obtener un resultado positivo por una técnica cuantitativa automatizada de inmunoanálisis. En este caso, se recomienda informar ambos resultados, aunque exista discrepancia entre ellos [37]. En general, no se considera adecuada la técnica de inmunotransferencia “blotting” para el análisis de anti-dsDNA [22].
Además, las guías reflejan que el informe debe incluir el método utilizado y los valores de referencia del laboratorio para los controles sanos y pacientes con LES [3, 37].

Consideran que en personas con clínica de LES, una prueba de anticuerpos antinucleares positiva y un título elevado de anti-dsDNA, el LES es la primera opción diagnóstica [36]. Una vez establecido el diagnóstico y para la monitorización de la actividad lúpica pueden emplearse técnicas cuantitativas; informándose los resultados cuantitativos y empleándose el mismo método durante el seguimiento, siempre que sea posible [37].

En nefritis lúpica activa, un estudio reciente compara métodos de Farr, ELISA, FEIA y CLIA; demostrando que Farr y FEIA poseen sensibilidades comparables del 95\%. Respecto a la actividad lúpica, Farr y FEIA se han comportado de forma similar [38].

\section{Estandarización}

El primer estándar internacional para anti-dsDNA, Wo/80 fue elaborado por la Organización Mundial de la Salud (WHO) en 1985 [39]. Este reactivo de referencia asignó Unidades Internacionales y mejoró la comparabilidad entre los test y los laboratorios; pero actualmente está agotado.

El Comité de expertos en estandarización y la WHO ha preparado y validado un nuevo reactivo de referencia, el denominado Reactivo de referencia anti-dsDNA (oligoespecífico) para lupus 15/174. Posee una potencia nominal de 100 unidades/ampolla; pero no es equivalente al primer estándar internacional (Wo/80) y no puede considerarse una continuidad del mismo. Está disponible en NIBSC (https:// www.nibsc.org/ products/ brm_ product_catalogue/detail_ page.aspx? catid=15/174) [40]. 


\section{Utilización clínica}

\section{Diagnóstico}

Los anticuerpos anti-dsDNA forman parte de los criterios de clasificación de LES, como puede observarse en la Tabla 2. En ella se presenta la definición y el peso relativo de los anti-dsDNA en cada uno de los criterios existentes [criterios ACR 1982, criterios ACR revisados 1997, criterios SLIIC 2012 y ACR-EULAR 2019 [41-44].

En los criterios SLICC 2012, los anti-dsDNA se analizaron mediante radioinmunoanálisis, CLIFT y ELISA. El criterio anti-dsDNA se asoció al diagnóstico de LES con una sensibilidad del 57,1\% y una especificidad del 95,9\%, sobre una muestra de 716 pacientes con diferentes enfermedades autoinmunes o inflamatorias [43]. En LES juvenil [en relación a artritis idiopática juvenil] la sensibilidad fue del 52,2\% y la especificidad del 100\% [45].

En los criterios ACR-EULAR 2019 [Tabla 3], 22 criterios se agrupan en diferentes dominios; aportándose un peso variable a cada criterio. Un paciente será clasificado de LES, cuando alcance 10 puntos y tenga, además, anticuerpos antinucleares positivos a título igual o superior a 1/80 en células HEp-2 o test equivalente. Los criterios y dominios se presentan en la tabla1 y no están limitados en el tiempo, pueden haber ocurrido previamente en el curso de la enfermedad [histórico de anticuerpos antinucleares

Tabla 2: Consideración de los anticuerpos anti-dsDNA en diferentes criterios de Lupus Eritematoso Sistémico.

\begin{tabular}{|c|c|c|}
\hline Criterio & Especificaciones & $\begin{array}{l}\text { Peso } \\
\text { relativo }\end{array}$ \\
\hline ACR $1982^{a}$ & Título anormal de anti-ADN nativo & $\begin{array}{l}1 / 11 \\
\text { criterios }\end{array}$ \\
\hline ACR $1997^{a}$ & Título anormal de anti-ADN nativo & $\begin{array}{l}1 / 11 \\
\text { criterios }\end{array}$ \\
\hline SLICC $2012^{b}$ & $\begin{array}{l}\text { Anti-dsDNA por encima del rango normal } \\
\text { del laboratorio, excepto ELISA: } \\
2 \text { veces por encima del rango normal de } \\
\text { referencia. }\end{array}$ & $\begin{array}{l}1 / 17 \\
\text { criterios }\end{array}$ \\
\hline $\begin{array}{l}\text { ACR-EULAR } \\
2018^{c}\end{array}$ & Anti-dsDNA de especificidad elevada & $\begin{array}{l}1 / 22 \\
\text { criterios }\end{array}$ \\
\hline
\end{tabular}

${ }^{a}$ Clasificación LES cuando: el paciente posee cuatro o más de un total de 11 criterios, seriados o simultáneos, durante un intervalo

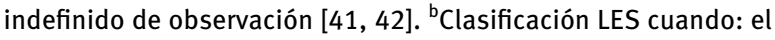
paciente satisface cuatro de los 17 criterios [11 clínicos y 6 inmunológicos] incluyendo al menos 1 criterio clínico y 1 inmunológico, de forma seriada o simultánea; o cuando el paciente tiene una nefritis confirmada por biopsia compatible con LES y anticuerpos antinucleares o anti-dsDNA positivos [43]. 'Clasificación de LES cuando: los anticuerpos antinucleares sean positivos a título $\geq 1 / 80$, la puntuación total sea $\geq 10$, se puntúe al menos un dominio clínico y dentro de cada dominio, se valore exclusivamente el criterio de mayor valor [Peso de anti-dsDNA: 6 puntos] [44]. positivo]. Los criterios no deben justificarse por patologías diferentes al LES y debe puntuarse al menos un dominio clínico. Cuando hay más de un criterio en un dominio clínico o inmunológico, solo se valorará el que tenga más peso. Los anti-dsDNA se integran en el dominio de anticuerpos altamente específicos. En su selección se tuvieron en cuenta las sensibilidades y especificidades de los criterios SLICC 2012 [referidos en el párrafo anterior] y ACR 1982 [67\% y 92\%, respectivamente] [41, 46].

\section{Asociación}

Numerosos trabajos han demostrado que los anti-dsDNA, son patogénicos en la nefritis lúpica [NL] y se asocian significativamente a ella [47]. Los anti-dsDNA ejercen su

Tabla 3: Criterios ACR/EULAR 2019 de Lupus Eritematoso Sistémico.

Dominios clínicos

Puntos

Dominio constitucional

Fiebre

Dominio cutáneo

Alopecia no cicatricial 2

Úlceras orales 2

Lupus cutáneo subagudo o discoide $\quad 4$

Lupus cutáneo agudo $\quad 6$

Dominio articular

Sinovitis o dolor en al menos dos articulaciones 6

$y>30$ minutos de rigidez articular

Dominio neurológico

Delirio

Psicosis

Convulsiones

3

Dominio de serositis

Derrame pleural o pericárdico 5

Pericarditis aguda

Dominio hematológico

Leucopenia

Trombocitopenia

Hemólisis autoinmune

Dominio renal

Proteinuria $>0.5 \mathrm{~g} / 24$ horas $\quad 4$

Nefritis lúpica de clase II ó V

Nefritis lúpica de clase III ó IV 10

Dominios inmunológicos

Puntos

Dominio de anticuerpos antifosfolípidos

aCL o aß2GP1 o anticoagulante lúpico

2

Dominio de proteínas del complemento

C3 o $\mathrm{C} 4$ bajas

3

C3 y C4 bajas

Dominio de anticuerpos altamente específicos

Anti-dsDNA

Anti-Sm

6

aCL, anti-cardiolipina; $a \beta 2 \mathrm{GP1}$, anti- $\beta 2$ glicoproteína1; anti-dsDNA, anti-DNA de doble cadena; anti-Sm, anti-Smith [41]. 
papel patogénico fijándose a lo largo de la membrana basal y matriz mesangial en forma de complejos inmunes circulantes formados por dsDNA y proteínas de la cromatina [48]; pero también, el anti-dsDNA circulante puede unirse directamente a estructuras renales (fragmentos de cromatina expuestos en la membrana basal o antígenos glomerulares con los que reacciona de forma cruzada). Estos dos mecanismos patogénicos pueden coexistir, e incluso predominar, uno $\mathrm{u}$ otro en las distintas fases de la nefritis lúpica $[49,50]$.

La asociación clínica con la nefritis lúpica se incrementa cuando los anti-dsDNA coexisten con anti-nucleosomas y anti-histonas; contribuyendo a diferenciarLES con NL de aquel sin NL y confiriendo severidad a la NL [51]. Otros anticuerpos que pueden asociarse a anti-dsDNA y NL son los anti-fracción-C1q del complemento y los anti-Sm [39]. Se ha descrito que la positividad de estos tres anticuerpos con concentraciones bajas de $\mathrm{C} 3 / \mathrm{C} 4 \mathrm{y}$ una relación disminuida albúmina/globulinas sugiere y parece predecir, afectación renal [52, 53]. Los más recientes, anticuerpos anti- proteínas de alta morbilidad que se unen al dsDNA parecen correlacionarse, igualmente, con anti-dsDNA y nefritis lúpica [53].

Algunas guías proponen monitorizar lar respuesta al tratamiento de la NL con niveles de anticuerpos antidsDNA y complemento, análisis del sedimento urinario, proteinuria en orina de 24 horas o cociente proteína/creatinina y creatinina sérica [54]. Sin embargo, otras han considerado que la especificidad de los anti-dsDNA con respecto a la NL es baja y poseen una utilidad limitada para el seguimiento de la misma [36].

\section{Seguimiento}

Las concentraciones de anti-dsDNA y componentes C3 y C4 [C3/C4] del complemento, actúan como marcadores serológicos de actividad en el LES. Así, y para la evaluación de la actividad, es adecuado evaluar conjuntamente el título de anticuerpos anti-dsDNA y los niveles de C3/C4 [55]. Si bien, hay que considerar, que la determinación de anticuerpos anti-dsDNA no va a predecir ni diagnosticar por sí solo un brote de actividad [3]. Los intervalos de medida se ajustarán a la situación clínica y serán, por tanto, variables. Si la enfermedad está en remisión clínica y analítica, se sugiere un seguimiento cada 6-12 meses, dependiendo del tiempo de evolución de la enfermedad y la intensidad del tratamiento. En pacientes clínicamente quiescentes, pero con criterios analíticos de actividad mantenidos, se sugiere un seguimiento más estrecho, cada 3-4 meses $[3,36,55]$.

Los niveles de $\mathrm{C} 3 / \mathrm{C} 4$ se modifican por el propio embarazo; no obstante, y a pesar de ello, se considera qué durante el embarazo, la determinación de $\mathrm{C} 3 / \mathrm{C} 4$ y antidsDNA permite monitorizar la actividad lúpica; debiendo realizarse su análisis ante sospecha de un brote de actividad [36]. Andreoli y cols, indican que la elevación de antidsDNA y la disminución de C3/C4 confieren a la gestante un riesgo aumentado de brote de LES [OR 5.3], al igual que incrementa el riesgo de pérdida fetal [56].

Los anticuerpos anti-dsDNA y C3/C4 están incluidos en índices clínicos de actividad del LES. En la Tabla 4 se presenta uno de los más utilizados, SLEDAI-2K descrito por Gladman [57] que recoge la presencia o ausencia de cada uno de los descriptores de actividad en los 30 días precedentes. SRI-50, basado en SLEDAI-2K, definemejoría clínica importante sobre el nivel basal al año, cuando los descriptores de SLEDAI-2K disminuyen $\geq 50 \%$ [58]. Existen otros índices de actividad como BILAG [British Isles Lupus AssessmentGroupIndex] y BILAG 2004, ECLAM [EuropeanConsensus Lupus ActivityMeasurement], SLAM [Systemic Lupus ActivityIndex]ySLAQ [SystemicLupus

Tabla 4: Índice de actividad de la enfermedad Lupus Eritematoso Sistémico [SLEDAI 2000] o SLEDAI-2K.

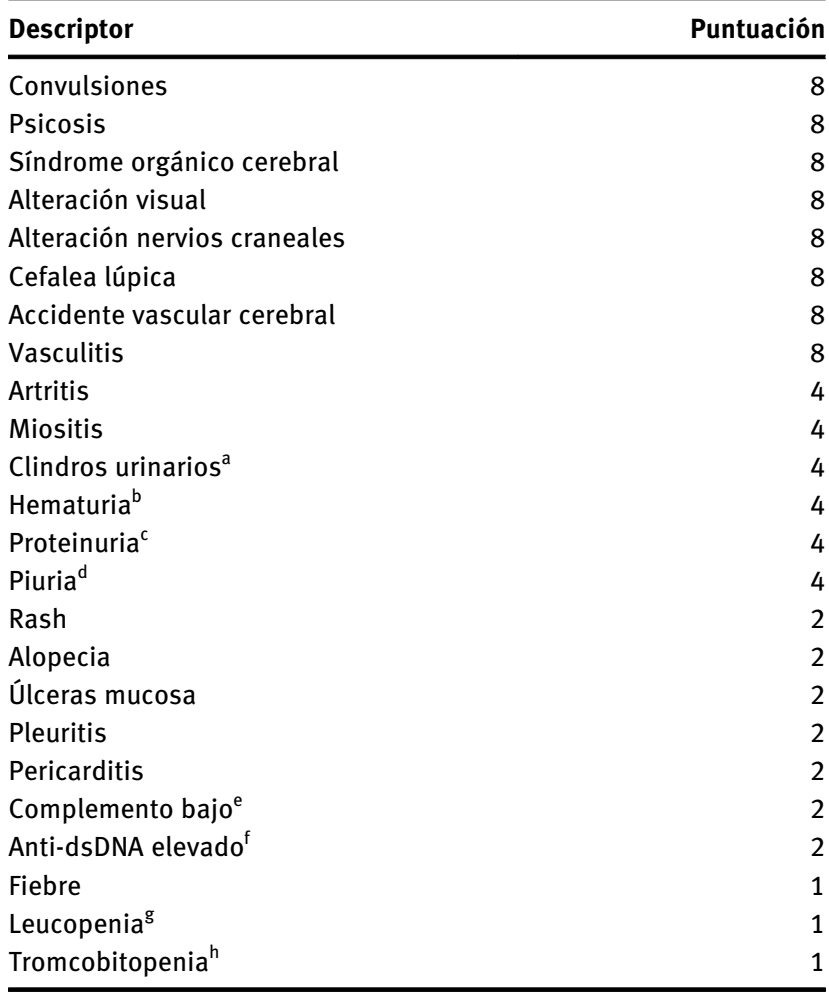

${ }^{a}$ Cilindros granulares o hemáticos; ${ }^{\mathrm{b}}$ más de 5 hematíes/campo; ${ }^{\mathrm{C}}$ más de $0,5 \mathrm{~g}$ proteínas $/ 24$ horas; ${ }^{\mathrm{d}}$ más de leucocitos/campo; e complemento $\mathrm{CH} 50, \mathrm{C} 3$ o C4; fanti-dsDNA superior al rango de referencia; ${ }^{\mathrm{g}}$ leucocitos $<3 \times 10^{9} / \mathrm{L}$; ${ }^{\mathrm{h}}$ plaquetas $<100 \times 10^{9} / \mathrm{L}$. Dependiendo del descriptor se considera reciente comienzo o persistencia. 
ActivityQuestionnaire] que no siempre van a considerar la elevación de anti-dsDNA y la hipocomplementemiaentre sus descriptores [59]. En 2016 un comité de expertos ha considerado, por consenso, la presencia de anti-dsDNA, junto con hipocomplementemia, como marcadores de actividad del LES (definidos como por encima o por debajo del valor de referencia del laboratorio). El 93\% de los expertos han recomendado añadir estos marcadores a la definición de remisión de LES [60].

Actualmente, se está intentando diferenciar actividad reducida y remisión del LES. Se tiende a considerar como remisión completa cuando el LES está inactivo sin medicación alguna o solo con anti-maláricos, como la hidroxicloroquina. Para actividad reducida existen diversas definiciones que tienen en cuenta SLEDAI-2K y los fármacos necesarios para mantener la actividad reducida. Se han definido, actividadmínima [MDA], actividad baja [LDA] y estado de actividad baja del LES [LLDAS]. La actividad serológica [medida con anti-dsDNA y C3/C4] está incluida exclusivamente en LLDAS. Mientras que MDA y LDA, solo tienen en cuenta variables clínicas [61].

En una guía reciente del Colegio Mexicano de Reumatología, se recomienda tomar en consideración los niveles bajos de complemento, la elevación de las concentraciones de anti-dsDNA y la elevación discreta de proteína $\mathrm{C}$ reactiva, para determinar en el paciente con LES, si la presencia de fiebre se asocia o no a la actividad de la enfermedad [54].

Para analizar la respuesta al tratamiento pueden emplearse distintos índices de respuesta [58]. No obstante, el papel de los anti-dsDNA en la monitorización del tratamiento es controvertido. Las experiencias son muy variables dependiendo de diversos factores, entre ellos los equipos de medida. Algunos expertos opinan que con una estandarizaran adecuada, bien solos o formando parte de un índice compuesto "score" con parámetros clínicos o analíticos, podrían monitorizar la respuesta al tratamiento (biológico o no-biológico) [1, 62, 63].

\section{Orientaciones de uso}

En este último apartado, los autores proponen orientaciones para una utilización clínica adecuada de los anti-dsDNA, derivadas de la información analizada.

El interés clínico y patogénico de los anti-dsDNA se incrementa cuando se detectan anti-dsDNA de afinidad y avidez elevadas. En general, se considera que se detectan anticuerpos alta avidez y afinidad con las técnicas de Farr [hoy, en desuso] y CLIFT, lo que les confiere una mayor especificidad. Por ello, se recomienda utilizar CLIFT en el diagnóstico de LES; si bien, puede utilizarse como técnica de segunda línea después de una técnica cuantitativa positiva. En caso de no poder realizar CLIFT, se considerarán resultados positivos los superiores a dos veces el límite superior del rango de referencia del grupo control. El clínico debe conocer el método utilizado y los rangos de referencia del laboratorio.

Para el diagnóstico y ante la sospecha clínica de un posible LES, los anti-dsDNA deben determinarse en pacientes con anticuerpos antinucleares positivos; informándose el resultado de ambos anticuerpos. Excepcionalmente, y ante una sospecha clínica elevada, podrían determinarse anti-dsDNA directamente; ya que pueden detectarse anti-dsDNA en pacientes con anticuerpos antinucleares negativos, en función de la técnica y el punto de corte utilizados; ya que influyen los métodos de fijación y las características de los métodos analíticos. Así, por ejemplo, en las células HEp-2, el dsDNA se encuentra en el núcleo unido a histonas y otras proteínas nucleares y se fija al soporte de vidrio con solventes orgánicos; mientras que en los métodos específicos, anti-dsDNA nativo, purificado o recombinante se une de forma aislada a un soporte sólido, generalmente plástico.

Los anti-dsDNA solo poseen utilidad diagnóstica en el LES y siempre deben interpretarse en el contexto clínico. Forman parte de los criterios de clasificación inmunológicos por su especificidad elevada. Una concentración elevada de anti-dsDNA en personas con clínica de LES y prueba de anticuerpos antinucleares positiva, sugiere LES como primera opción diagnostica; sin embargo, un resultado negativo no va a excluir LES, dada su pobre sensibilidad y razón de verosimilitud negativa.

Se utilizará la determinación de anti-dsDNA cuantitivo para el seguimiento del paciente con LES, recomendándose emplear el mismo método en todo el seguimiento.

Los anti-dsDNA se asocian a actividad lúpica, especialmente cuando se detectan a concentraciones elevadas, con métodos de especificidad elevada y se asocian a algún otro signo clínico o de laboratorio de actividad en el paciente. Una elevación de anti-dsDNA no predice ni diagnostica por sí sola una activación de la enfermedad.

Los anti-dsDNA y los niveles de $\mathrm{C} 3 / \mathrm{C} 4$ del complemento están incluidos en índices de actividad de LES y se recomienda su evaluación conjunta ante la sospecha de un brote de actividad, incluidas gestantes con LES.

Los anti-dsDNA se correlacionan con NL y poseen una utilidad relativa para su diagnóstico y actividad. Al igual que con la actividad, concentraciones y especificidades elevadas, mejoran la asociación y la utilidad clínica de los anti-dsDNA en NL; pero deben interpretarse en conjunción con otras medidas de enfermedad renal. 


\section{Conclusiones}

Existe una amplia variabilidad en los métodos analíticos empleados clínicamente para el análisis de anti-dsDNA; asî como déficits en su estandarización. No todos los métodos van a detectar anticuerpos con el mismo grado de avidez y afinidad. Los anti-dsDNA de avidez y afinidad elevadas en pacientes con ANA positivos, poseen una elevada especificidad diagnóstica para LES. En el seguimiento, es interesante la cuantificación de estos anticuerpos, preferiblemente con el mismo método analítico (dado los déficits de estandarización). Destacar, por último, que la variabilidad y los avances en los métodos de medida van modificando sus características clínicas.

Financiación de la investigación: No declarada. Contribución de los autores: Todos los autores han aceptado la responsabilidad del contenido completo del manuscrito ya prueban su envoi.

Conflicto de intereses: Los autores declaran no tener ningún conflicto de intereses.

\section{Referencias}

1. Bragazzi NL, Watad A, Damiani G, Adawi M, Amital H, Schoenfeld Y. Role of anti-DNA autoantibodies as biomarkers of response to treatment in systemic lupus erythematosus patients: hypes and hopes. Insights and implications form a comprehensive review of the literature. Expert Rev Mol Diagn 2019;19:969-78.

2. Mummert E, Fritzler MJ, Sjöwall C, Bentow C, Mahler M. The clinical utility of anti-double-stranded DNA antibodies and the challenges of their determination. J Immunol Methods 2018;459:11-19.

3. Kavanaugh AF, Solomon DH, the American College of Rheumatology ad hoc Committee on Immunologic Testing Guidelines. Guidelines for immunologic laboratory testing in the rheumatic diseases: anti-DNA antibody tests. Arthritis Rheum 2002;47:546-55.

4. Kornberg RD. Chromatin structure: a repeating unit of histones and DNA. Science 1974;184:868-71.

5. Pisetsky DS. Anti-DNA antibodies - quintessential biomarkers of SLE. Nat Rev Rheumatol 2016;12:102-10.

6. Gómez-Puerta JA, Burlingame RW, Cervera R. Anti-chromatin (AntiNucleosome) antibodies: diagnostic and clinical value. Autoimmun Rev 2008;7:606-11.

7. Ghiggeri GM, D'Alessandro M, Bartolomeo D, Degl'Innocenti ML, Magnasco A, Lugani F, et al. An update on antibodies to nucleosome components as biomarkers of sistemic lupus erythematosus and of lupus flares. Int J Mol Sci 2019;20:5799.

8. Charavi AE, Reiver H. Affinity and avidity of autoantibodies. Autoantibodies 1996:13-23. https://doi.org/10.1016/b978044482383-0/50005-4.

9. Wang JJ, Colella AD, Beroukas D, Chataway TK, Gordon TP. Precipitating anti-dsDNA peptide repertoires in lupus. Clin Exp Immunol 2018;194:273-82.
10. Rekvig OP. Systemic lupus erythematosus: definitions, contexts, conflicts, enigmas. Front Immunol 2018;9:387.

11. Hamilton KJ, Schett G, Reich CF III, Smolen JS, Pisetsky DS. The binding of sera of patients with SLE to bacterial and mammalian DNA. Clin Immunol 2006;118:209-18.

12. Rekvig OP. The anti-DNA antibody: origin and impact, dogmas and controversies. Nat Rev Rheumatol 2015;11:530-40.

13. Akberova NI, Zhmurov AA, Nevzorova TA, Litvinov RI. An anti-DNA antibody prefers damaged dsDNA over native. J Biomol Struct Dyn 2017;35:219-23.

14. Kavai M, Banyai A, Zsindely A, Sonkoly I, Szeged G. Enzymelinked immunosorbent assay for antibodies to native DNA in sera of patients with SLE. J Immunol Methods 1982;48:169-75.

15. Aarden LA, Lakmaker F, de Groot ER, Swaak AJ, Feltkamp TE. Detection of antibodies to DNA by radioimmunoassay and immunofluorescence. Scand J Rheumatol Suppl 1975;b11:12-9.

16. Pincus T, Schur PH, Rose JA, Decker JL, Talal N. Measurement of serum DNA-binding activity in systemic lupus erythematosus. N Engl J Med 1969;281:701-5.

17. Hernando M, Gonzalez C, Sanchez A, Guevara P, Navajo JA, Papisch $W$ et al. Clinical evaluation of a new automated anti-dsDNA fluorescent immunoassay. Clin Chem Lab Med 2002;40:1056-60.

18. Bentow C, Lakos G, Martis P, Wahl E, Garcia M, cols VOy. International multi-center evaluation of a novel chemiluminescence assay for the detection of anti-dsDNA antibodies. Lupus 2016;25:864-72.

19. Shovman O, Gilburd B, Barzilai O, Shinar E, Larida B, ZandmanGoddard G, et al. Evaluation of the BioPlex 2200 ANA screen: analysis of 510 healthy subjects: incidence of natural/predictive autoantibodies. Ann N YAcad Sci 2005;1050:380-8.

20. Shovman O, Gilburd B, Zandman-Goddard G, Yehiely A, Langevitz P, Shoenfeld Y. Multiplexed Athenha multi-lyte immunoassay for ANA screening in autoimmune diseases. Autoimmunity 2005;38:105-9.

21. UK-NEQAS international quality experti antibodies to nuclear and related antigens program. Available from: https://www.immqas. org.uk/ [Consultado 25 June 2019].

22. Van Blerk M, Bossuyt X, Humbel R, Mewis A, Servais G, Tomasi JP, et al. Belgian recommendations on ANA, anti-dsDNA and anti-ENA antibody testing. Acta Clin Belg 2014;69:83-6.

23. Bernard JF, Hockley J, Risgby P, Dolman C, Meroni PL, Rönnelid J. A WHO Reference Reagent for lupus (anti-dsDNA) antibodies: international collaborative study to evaluate a candidate preparation. Ann Rnheum Dis 2019;78:1677-80.

24. Farr RSJ, Minden, Anthony BF. A comparison of seven procedures to detect the primary binding of antigen by antibody. J Immunol 1969;102:832-41.

25. MahlerM, Fritzler MJ. Anti-dsDNA antibody testing in the clinic: farr or ELISA? Nat Clin Pract Rheumatol 2007;3:72-3.

26. Lakota K, Švec T, Kveder T, Sodin-Šemrl S, Žigon P, Ambrožič A, et al. Autoantibodies against dsDNA measured with nonradioactive farr assay-an alternative for routine laboratories. Clin Rheumatol 2019;38:353-9.

27. Compagno M, Jacobsen S, Rekvig OP, Truedsson L, Heegaard NH, Nossent J, et al. Low diagnostic and predictive value of anti-dsDNA antibodies in unselected patients with recent onset of rheumatic symptoms: results from a long-term follow-up Scandinavian multicentre study. Scand J Rheumatol 2013;42:311-6.

28. Tan ZJ, Chen SJ. Nucleic acid helix stability: effects of salt concentration, cation valence and size and change length. Biophys J 2006;90:1175-90. 
29. Infantino M, Meacci F, Bentow C, Martis P, Benucci M, Afeltra A, et al. Clinical comparison of QUANTA Flash dsDNA chemiluminescent immunoassay with four current assays for the detection of anti-dsDNA autoantibodies. J Immunol Res 2015; 2015:902821.

30. Abuaf N, Desgruelles C, Moumaris M, Boussa-Khettab F, Rostane $\mathrm{H}$, Bellec E, et al. Detection by flow cytometry of anti-DNA autoantibodies and circulating DNA immune complexes in lupus erythematosus. J Immunol Res 2019:6047085. https://doi.org/ $10.1155 / 2019 / 6047085$.

31. Ghillani P, Dufat L, Hilmeur S, Miyara M, Amoura Z, Musset L. Routine use of Zenit RA, a novel chemiluminescent immunoanalyzer in autoimmune disease diagnosis. Auto Immun Highlights 2012;21:27-31.

32. Liping GY, Wang J, O'Dell JR, Oates J, Arend WP, Eisenbarth GS. Anti-dsDNA antibody assay: high specificity and sensitivity with a filtration radioassay in comparison to low specificity with the standard ELISA. J Rheumatol 2007;34:734-9.

33. Infantino M, Manfredi M, Merone M, Grossi V, Benuci M, Gobbi FL, et al. Analytical variability in the determination of anti-double stranded DNA antibodies: the strong need of a better definition of the old and new tests. Immunol Res 2018;66:340-7.

34. Enocsson H, Sjöwall C, Wirestam L, Dahle C, Kastbom A, Rönnelid J, et al. Four anti-dsDNA antibody assays in relation of systemic lupus erythematosus disease specificity and activity. J Rheumatol 2015;42: $817-5$.

35. Ghirardello A, Villalta D, Morozzi G, Afeltra A, Galeazzi M, Gerli R, et al. Evaluation of current methods for the measurement of serum anti double-stranded DNA antibodies. Ann N Y Acad Sci 2007;1109:401-6.

36. Grupo de trabajo sobre la guía de práctica clínica sobre lupus eritematoso sistémico. Guía de práctica clínica sobre lupus eritematoso sistémico. Ministerio de Sanidad, Servicios Sociales e Igualdad. Servicio de Evaluación del Servicio Canario de Salud. Guías de Práctica Clínica en el SNS; 2015.

37. Agmon-Levin N, Damoiseaux J, Kallenberg C, Sack U, Witte T, Herold $\mathrm{M}$, et al. International recommendations for the assessment of autoantibodies to cellular antigens referred to as anti-nuclear antibodies. Ann Rheum Dis 2014;73:17-23.

38. de Leeuw K, Bungener L, Roozendaal C, Bootsma H, Stegeman CA. Auto-antibodies to double-stranded DNA as biomarker in systemic lupus erythematosus: comparison of different assays during quiescent and active disease. Rheumatology 2017;56:698-703.

39. Feltkamp TE, Kirkwood TB, Maini RN, et al. The first international standard for antibodies to double stranded DNA. Ann Rheum Dis 1988;47:740-6.

40. Fox BJ, Hockley J, Rigsby P, Dolman C, Meroni PL, Rönnelid J. A WHO Reference Reagent for lupus (anti-dsDNA) antibodies: international collaborative study to evaluate a candidate preparation. Ann Rheum Dis 2019;78:1677-80.

41. Tan EM, Cohen AS, Fries JF, Masi AT, McShane DJ, Rothfield NF, et al. The 1982 revised criteria for the classification of systemic lupus erythematosus. Arthritis Rheum 1982;25:1271-7.

42. Hochberg MC. Updating the American College of Rheumatology revised criteria for the classification of systemic lupus erythematosus. Arthritis Rheum 1997;40:1725.

43. Petri M, Orbai AM, Alarcón GS, Gordon C, Merrill JT, Fortin PR, et al. Derivation and validation of the Systemic Lupus International Collaborating Clinics classification criteria for systemic lupus erythematosus. Arthritis Rheum 2012;64:2677-8.
44. Aringer M, Costenbader K, Daikh D, Brinks R, Mosca M, Ramsey-Goldman $R$, et al. European League against Rheumatism/American College of Rheumatology classification criteria for systemic lupus erythematosus. Annals Rheum Dis 2019;78:1151-9.

45. Osaku FM, Fraga MM, Aires PP, Caparroz AA, Len CA, Terreri MT. SLICC classification criteria for juvenile systemic lupus erythematosus: a cross sectional study. Acta Reumatol Port 2018; 43:279-83.

46. Tedeschi SK, Johnson SR, BouMPIAs D, Daikh D, Dörner T, Jayne D, et al. Developing and refining new candidate criteria for SLE classification: an international collaboration. Arthritis Care Res 2018;70:571-81.

47. Anders H-J, Rovin B. A pathophysiology-based approach to the diagnosis and treatment of lupus nefritis. Kidney Int 2016;90: 493-501.

48. Van derVlag V, Berden JH. Lupus nephritis: role of antinucleosome autoantibodies. Semin Nephrol 2011;31:376-89.

49. Fenton K, Fismen S, Hedberg A, Seredkina N, Fenton C, Mortensen ES, et al. Anti-dsDNA antibodies promote initiation, and acquired loss of renal Dnase1 promotes progression of lupus nephritis in autoimmune (NZBxNZW)F1 mice. PloS One 2009;4:e8474.

50. Rekvig OP. The dsDNA, anti-dsDNA antibody, and lupus nephritis: what we agree on, what must Be done, and what the best strategy forward could Be. Front Immunol 2019;10:1104.

51. Yang J, Xu Z, Sui M, Han J, Sun L, Jia X, et al. Co-Positivity for AntidsDNA, -nucleosome and -histone antibodies in lupus nephritis is indicative of high serum levels and severe nephropathy. PloS One 2015;10:e0140441.

52. Kwon OC, Lee JS, Ghang B, Kim Y-G, Lee C-K, Yoo B, et al. Predicting eventual development of lupus nephritis at the time of diagnosis of systemic lupus erythematosus. Semin Arthritis Rheum 2018;48:462-6.

53. Choi MY, Fritzler MJ. Challenges and advances in SLE autoantibody detection and interpretation. Curr Treat Options Rheum 2019;5:147-7.

54. Xibillé-Friedmann, Pérez-Rodríguez $M$, Carrillo-Vázquez $\mathrm{S}$, Álvarez-Hernández E, Aceves FJ, Ocampo-Torres MC. Guía de práctica clínica para el manejo del lupus eritematoso sistémico propuesta por el Colegio Mexicano de Reumatología. Reumatol Clínica 2019;15:3-20.

55. Alarcón Torres I, González Rodríguez C, Jiménez Jiménez J, Fernándes Suárez A, Alsina Donadeu M. Actualización del manejo de los anticuerpos antinucleares en las enfermedades autoinmunes sistémicas. SEQC ${ }^{\mathrm{ML}}$ Ed. Documentos de la SEQC 2014;7:73-84.

56. Andreoli L, Bertsias GK, Agmon-Levin N, Brown S, Cervera R, Costedoat-Chalumeau N, et al. EULAR recommendations for women's health and the management of family planning, assisted reproduction, pregnancy and menopause in patients with systemic lupus erythematosus and/or antiphospholipid syndrome. Ann Rheum Dis 2017;76:476-85.

57. Gladman DD, Ibáñez D, Urowitz MB. Systemic lupus erythematosus disease activity index 2000. J Rheumatol 2002;29:288-9.

58. Touma Z, Gladman DD, Ibañez D, Urowitz MB. Development and initial validation of the systemic lupus erythematosus disease activity index 2000 responder index 50. J Rheumatol 2011;38:275-84.

59. Castrejón I, Tani C, Jolly M, Huang A, Mosca M. Indices to assess patients with systemic lupus erythematosus in clinical trials, 
long-term observational studies, and clinical care. Clin Exp Rheumatol 2014;32:S85-95.

60. Van Vollenhoven R, Voskuyl A, Bertsias G, Aranow C, Aringer $M$, Arnaud L, et al. A framework for remission in SLE: consensus findings from a large international task force on definitions of remission in SLE (DORIS). Ann Rheum Dis 2017;76:554-61.

61. Tselios K, GladmanDD, Urowitz MB. How can define low disease activity in systemic lupus erythematosus? Sem Arthritis Rheum 2018;48:1035-40.

62. Bragazzi NL, Watad A, Damiani G, Adawi M, Amital H, Shoenfeld Y. Role of anti-DNA auto-antibodies as biomarkers of response to treatment in systemic lupus erythematosus patients: hypes and hopes. Insights and implications from a comprehensive review of the literature. Expert Rev Mol Diagn 2019;19:969-78.

63. Pisetsky DS, Rovin BH, Lipsky PE. New perspectives in rheumatology: biomarkers as entry criteria for clinical trials of new therapies for systemic lupus erythematosus: the example of antinuclear antibodies and anti-DNA. Arthritis Rheumatol 2017;69:487-93.

Nota del artículo: La versión traducida del artículo puede encontrarse aquí: https://doi.org/10.1515/almed-2021-0008. 